\title{
Discussion and future perspectives
}

The discovery of small RNAs like miRNAs has revolutionized the concept of genes and gene regulation. It is clear that miRNAs play an important role in virtually all biological processes and that their dysregulation is associated with disease. In cancer, widespread changes in miRNAs levels have been observed in virtually all tumor types. Moreover, mouse studies have demonstrated that causal links exist between miRNAs and cancer development [1-4], and miRNAs have been demonstrated to play crucial roles in the response to therapy [5-6].

Given the current interest in miRNAs it is likely that in the following years the role of miRNAs in tumor biology will be better understood. It is likely that in the future the focus will shift from identifying individual miRNAs to describing miRNA networks in healthy and cancer cells [7-10]. High throughput sequencing will enable comprehensive and quantitative analysis of existing and novel miRNAs (as well as different miRNA isoforms) in cancer and normal tissues. However, these approaches generate a lot of data, and the challenge will be to filter out the miRNAs that play a central role in cancer biology. The method we describe in Chapter 2 may help to identify those key miRNAs. We demonstrated that tumor miRNAs that are regulated after DNA damage in primary breast and lung epithelial cells are more likely to modulate the response to genotoxic agents. This method can be extended to other forms of cancer as well. Further studies are necessary to determine whether the regulation of the DDR miRNAs is directly mediated by ATM/ATR signaling and whether these miRNAs affect regulation of the cell cycle and/or repair of DNA lesions. 
We have developed an assay to measure the effects of miRNA overexpression on cancer drug sensitivity (Chapter 2, Chapter 5-7). This assay could be used to screen for other miRNAs that play a role in cancer drug sensitivity. In this thesis we have specifically focused on the role of miR-141 and miR-634 in the response to platinum-based chemotherapy in ovarian cancer. Avenues for future research and therapeutic options for these miRNAs will be discussed in the remainder of this chapter.

\section{The miR-200 family of miRNAs}

The miR-200 family consists of 5 miRNAs: miR-200a,b,c, miR-141 and miR429 (Chapter 4). In Chapter 5 we demonstrated that miR-141 can induce cisplatin resistance in ovarian cancer cell lines. However, the miR-200 family has also been associated with the response to paclitaxel, docetaxel, gemcitabine, doxorubicin, imatinib, tamoxifen and erlotinib/gefitinib chemotherapy (Table 1) [11-21]. These drugs have different modes of action. Whereas cisplatin and doxorubicin interact with the DNA [22-23], paclitaxel and docetaxel inhibit microtubules and stimulate the production of ROS [24-26] and gemcitabine is a nucleoside analogue that interferes with DNA synthesis [27]. Imatinib, erlotinib and gefitinib are tyrosine kinase inhibitors [28-29], whereas tamoxifen binds to the estrogen receptor and interferes with estrogen receptor signaling [30]. The question arises how the miR-200 family can modulate the sensitivity towards these different kinds of drugs.

An explanation for this could be that the miR-200 family inhibits the Epithelialto-Mesenchymal transition (through repression of ZEB1, ZEB2 (all members) and BMI1 (miR-200b) [31-34]; see Chapter 4). 
Table 1: $m i R-200$ family and response to anticancer drugs

\begin{tabular}{|c|c|c|c|c|c|}
\hline Cytotoxic agent & Tumor type & miR-200 family member & Association & Potential target & Reference \\
\hline Cisplatin & Esophageal cancer & $\operatorname{miR}-141$ & High levels confer resistance to cisplatin & \multirow{2}{*}{$\begin{array}{l}\text { YAP1 } \\
\text { PPP2R1B, correlation } \\
\text { only }\end{array}$} & [35] \\
\hline Cisplatin & Esophageal cancer & miR-200c & High levels confer resistance to cisplatin & & [18] \\
\hline Cisplatin & Ovarian cancer & $\operatorname{miR}-141$ & $\begin{array}{l}\text { High levels confer resistance towards } \\
\text { cisplatin }\end{array}$ & \multirow[t]{3}{*}{ KEAP1 } & Chapter 5 \\
\hline Cisplatin & Ovarian cancer & $m i R-141$ and $m i R-200 c$ & $\begin{array}{l}\text { High levels correlate with resistance in a } \\
\text { subset of patients }\end{array}$ & & [36] \\
\hline Cisplatin & Breast cancer & $m i R-141, m i R-200 b, m i R-200 c$ & \multirow{2}{*}{$\begin{array}{l}\text { Expression lower in resistant cells } \\
\text { Low levels confer resistance towards } \\
\text { cisplatin }\end{array}$} & & [37] \\
\hline Cisplatin & Tongue Squamous Cell Carcinoma & miR-200b & & \multirow{4}{*}{$\begin{array}{l}\text { BMI1 } \\
T U B B 3, \text { correlation only } \\
T \cup B B 3\end{array}$} & [31] \\
\hline Paclitaxel & Endometrium and ovarian cancer & miR-200c & High levels sensitize towards paclitaxel & & [11] \\
\hline Paclitaxel & Endometrium and ovarian cancer & miR-200c & High levels sensitize towards paclitaxel & & [12] \\
\hline Paclitaxel & Ovarian cancer & All & High levels sensitize towards paclitaxel & & [38] \\
\hline Paclitaxel & Ovarian cancer & $m i R-200 a$ and $m i R-141$ & High levels sensitize towards paclitaxel & p38 MAPK & [13] \\
\hline Docetaxel & Lung cancer & miR-200b & High levels sensitize towards docetaxel & \multirow[t]{2}{*}{ E2F3 } & [14] \\
\hline Docetaxel & Lung cancer & $m i R-200 b$ & Expression lower in resistant cells & & [39] \\
\hline Doxorubicin & Breast cancer & $m i R-141$ & Expression higher in resistant cells & & [17] \\
\hline Doxorubicin & Breast cancer & $m i R-200 b$ & High levels sensitize towards doxorubicin & & [40] \\
\hline Doxorubicin & Breast cancer & miR-200c & Expression lower in resistant cells & & [41] \\
\hline Doxorubicin & Lung cancer & miR-200b & Expression lower in resistant cells & & [42] \\
\hline Erlotinib/Gefitinib & Bladder cancer & miR-200c & $\begin{array}{l}\text { High levels sensitize towards } \\
\text { Erlotinib/Gefitinib }\end{array}$ & ERRFI1 & [19] \\
\hline Gemcitabine & Cholangiocarcinoma & $m i R-141$ and $m i R-200 b$ & $\begin{array}{l}\text { High levels confer resistance towards } \\
\text { gemcitabine }\end{array}$ & \multirow[t]{3}{*}{$\begin{array}{l}\text { miR-141 - Clock, miR- } \\
200 b-P T P N 12 \\
\text { (correlation only) }\end{array}$} & [15] \\
\hline Gemcitabine & Pancreatic cancer & $\mathrm{miR}-200 \mathrm{~b} / \mathrm{c}$ & Expression lower in resistant cells & & [43] \\
\hline Gemcitabine & Pancreatic cancer & $\mathrm{miR}-200 \mathrm{~b} / \mathrm{c}$ & Expression lower in resistant cells & & {$[16]$} \\
\hline Imatinib & Chronic Myeloid Leukemia & $\operatorname{miR}-141$ & Expression lower in resistant cells & & {$[20]$} \\
\hline Tamoxifen & Breast cancer & $m i R-200$ & Expression lower in resistant cells & & [21] \\
\hline
\end{tabular}


In general, mesenchymal cells are more drug resistant than epithelial cells [44], and by converting mesenchymal cells to the epithelial state, miR-200 family overexpression may enhance drug sensitivity. In addition, miR-200 members downregulate targets that play a role in cell proliferation and survival, making cells more sensitive for death inducing stimuli. These targets include the cell cycle regulator E2F3 (miR-200b) [14] and the stress response gene p38 MAPK (miR-200a, miR-141) [13]) (Table 1).

However, regulation of these targets is not likely to account for the decreased sensitivity of cancer cells to cisplatin upon overexpression of miR-141. Downregulation of YAP1, a protein that plays a role in DNA damage induced apoptosis [45] (Figure 1) may contribute to miR-141-mediated cisplatin resistance [35]. Furthermore, we demonstrated in Chapter 5 that the phenotype of miR-141 is partially mediated by repression of KEAP1, a key regulator of the NRF2-mediated oxidative stress response, the NF- $\mathrm{kB}$ pathway and the anti-apoptotic protein $\mathrm{Bcl}-\mathrm{xL}$ [46-50]). Under normal homeostatic conditions KEAP1 acts as a repressor of NRF2, IKK $\beta$ (activator of NF-KB) and $\mathrm{Bcl}-\mathrm{xL}$, however, under stress KEAP1 becomes inactivated resulting in derepression. Downregulation of KEAP1 by miR-141 may thus also lead to activation of these pathways, providing a molecular basis for miR-141 induced cisplatin resistance. Accordingly, we showed that miR-141 overexpression results in activation of NF-kB signaling, presumably through repression of KEAP1 and activation of IKK [48-49] (Figure 1). More research is necessary to investigate why KEAP1 downregulation does not result in activation of the NRF2 pathway. It will also be interesting to investigate if activation of NF-kB by miR-141 affects the response to other chemotherapeutics. Of note, paclitaxel and doxorubicin treatment activate NF-кB signaling [51-54]. 
It will also be interesting to look into the mechanisms that regulate miR-200 expression in response to cisplatin treatment (Chapter 5). It has been reported that p53, a key mediator of the cellular response to DNA damage, can induce expression of miR-141 and other members of the miR-200 family [55-56], and overexpression of miR-141 results in downregulation of p53 [57]. The relation between p53 and miR141 signaling in the cellular response to cytotoxics needs to be further elucidated.

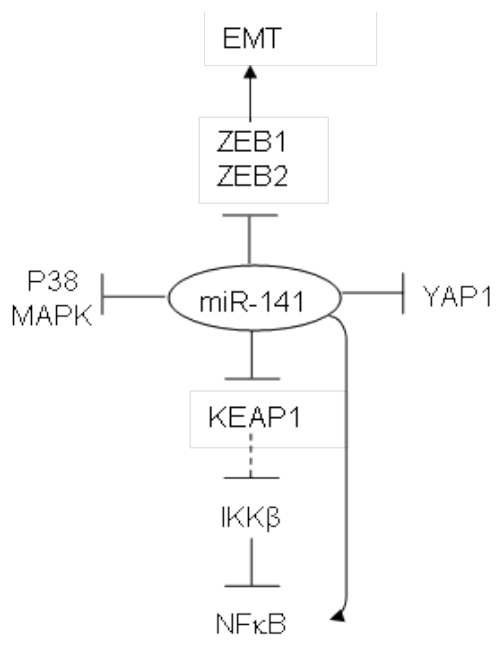

Figure 1: Pathways regulated by miR-141 that may affect the response to anticancer therapy

miR-141 has been reported to inhibit the EMT regulators ZEB1/ZEB2 [32-34] and p38 $\alpha$ MAPK $[7,13]$. Repression of these targets may result in enhanced chemotherapy sensitivity. Furthermore, repression of YAP1 [7, 35] and KEAP1 (Chapter 5) may enhance resistance. KEAP1 levels may modulate sensitivity to platinum-containing chemotherapeutics (e.g. cisplatin) by regulating the NF-KB pathway. Accordingly, miR-141 overexpression results in NF-KB activation and inhibition of NF-KB partially reverses the miR-141 phenotype. However, further experiments are necessary to demonstrate that these effects are mediated by KEAP1 and IKK $\beta$.

\section{2. $m i R-634$}

In contrast to the miR-200 family, miR-634 has not received a lot of attention. The miRNA was first identified in colon cancer cells [58], and afterwards a study appeared where it was demonstrated that miR-634 targets the androgen receptor (AR) [59]. Of note, high androgen serum levels are correlated with increased ovarian cancer risk [60] and more than $80 \%$ of ovarian cancer tumors express the AR [61].

In chapter 6 we describe that overexpression of miR-634 results in increased sensitivity towards cisplatin, carboplatin and doxorubicin, but not paclitaxel. Paclitaxel 
can promote cell death via at least two mechanisms (i) the inhibition of microtubule dynamics leading to a G2/M arrest [24] and (ii) stimulation of the production of ROS [25-26]. Because the drug exposure time in our assay is only 24 hours (and the population doubling time of the ascites derived cultures is $\sim 48$ hours or less) it is conceivable that the effects of paclitaxel on the cell cycle do not contribute to the reduction in viability. Therefore, more studies are necessary to investigate the effect of miR-634 on paclitaxel cytotoxicity.

We proposed in Chapter 6 that the effects of miR-634 on drug sensitivity are due to regulation of several components of the Ras-MAPK pathway. In Chapter 7 we further show that repression of one of its predicted targets RSK2, results in enhanced cisplatin sensitivity in ovarian cancer cell lines. In contrast, knockdown of the putative miR-634 target and related kinase RSK1, does not alter cisplatin sensitivity. The fact that RSK2, but not RSK1, is downregulated in response to cisplatin treatment may indicate that this protein plays an important role in the cellular response to cisplatin treatment. The importance of the RSK family in the response to cisplatin treatment is highlighted by the fact that another recent study found that RSK expression levels correlate with cisplatin resistance in a panel of 8 ovarian cancer cell lines [62].

Intriguingly, the predicted targets of miR-634 are enriched for genes that play a role in prostate cancer, acute myeloid leukemia, glioma, non-small cell lung cancer, endometrial cancer, clear cell renal cancer, thyroid cancer, colorectal cancer and pancreatic cancer (Supplementary table 4, Chapter 6). Therefore, it will be interesting to study the effect of miR-634 overexpression on these tumors. Of note, ascites formation is also observed in patients with advanced cancers of other organs in the abdominal cavity, such as lung cancer, colorectal cancer, and pancreatic cancer [63]. 
Hence, a first step may be to analyze the effects of miR-634 overexpression on ascites-derived tumor cells in these patients.

\section{Towards a miRNA-based therapy}

A decade after its discovery miRNAs are rapidly entering the clinic as biomarkers and therapeutic tools [64-65]. The fact that miRNAs can regulate the expression of multiple genes makes them attractive drug targets, as multiple oncogenes can be inhibited at the same time. However, the fact that miRNAs can regulate multiple genes is also a major drawback, because of the risk of unwanted side effects. To reduce side effects, tumor-specific delivery may be necessary.

Several studies have demonstrated that a miRNA-based therapy is feasible. Oncogenic miRNAs can be inhibited by anti-miRNA oligonucleotides (e.g. antagomirs or Locked Nucleic Acids [66-67]) or via constructs containing miRNA target sequences ('sponges' or 'decoys' [68-69]). Tumor-suppressive miRNAs may be repleted by synthetic miRNA molecules or via expression constructs [70-73] Delivery of (anti) miRNAs by 'naked' synthetic oligonucleotides [67, 74-80], oligonucleotides packaged in protein- or lipid based nanocapsules [81-84] or by viral particles [72, 8587].

Ovarian cancer represents a group of heterologous tumors with different etiology. Since the high grade serous ovarian cancer is the most common histotype and accounts for $70 \%$ of the deaths [88], for a miR-based therapy it may be best to focus on this subtype. There is currently only one mouse model that recapitulates the development of human high grade serous ovarian cancer, however, this model is based on knockout of Pten and Dicer [88]. Since Dicer is required for miRNA biogenesis, studies may be best performed in human ovarian tumor xenografts [89]. 
To direct expression of miRNAs to ovarian tissue, viral-mediated delivery is probably the best option. Both lentiviral as adenoviral vectors could be used. Lentiviral vectors stably integrate into the genome of the host cell [90], but a disadvantage is that they can disrupt genes. Adenoviral constructs $[70,72]$ are not incorporated into the DNA of the host cell, but the effect of viral transfection is transient. The tropism of viruses may be altered so that they may bind to cell type specific receptors [91-92]. To direct viruses specifically to ovarian tumor cells the folate receptor (which is expressed in $90 \%$ of ovarian tumor cells) may be targeted [93]. Furthermore, fallopian tube specific transcription factors (e.g. Pax8) may be used to confine expression to tumor cells [94].

As a first step towards resensitizing ovarian tumors to chemotherapy, the effects of miR-634 overexpression on the therapy response of xenograft tumors could be determined. Before miR-141 can progress towards the clinic, the effect of miR-141 overexpression on the outcome of combination therapy (ovarian cancer is treated with a platinum-based compound and a taxane) needs to be better understood. miR141 can both sensitize ovarian cancer cells for paclitaxel and enhance cisplatin resistance and therefore overexpression may differentially affect the outcome of treatment (Chapter 5, [11-13, 36, 38]. Next to use as a therapeutic target, miR-141 and miR-634 may also be exploited as biomarker to predict the response of tumors to chemotherapy.

Altogether, therapies based on miR-141 and miR-634 hold promise for the treatment of ovarian cancer.

1. Costinean, S., et al., Pre-B cell proliferation and lymphoblastic leukemia/high-grade lymphoma in $E(m u)-m i R 155$ transgenic mice. Proc Natl Acad Sci U S A, 2006. 103(18): p. 7024-9. 
2. Medina, P.P., M. Nolde, and F.J. Slack, OncomiR addiction in an in vivo model of microRNA21-induced pre-B-cell lymphoma. Nature, 2010. 467(7311): p. 86-90.

3. He, L., et al., A microRNA polycistron as a potential human oncogene. Nature, 2005. 435(7043): p. 828-33.

4. Ma, L., J. Teruya-Feldstein, and R.A. Weinberg, Tumour invasion and metastasis initiated by microRNA-10b in breast cancer. Nature, 2007. 449(7163): p. 682-8.

5. Zhang, W. and M.E. Dolan, The emerging role of microRNAs in drug responses. Curr Opin Mol Ther, 2010. 12(6): p. 695-702.

6. Wiemer, E.A.C., Role of MicroRNAs in Anti-cancer Drug Resistance, in MicroRNAs in Cancer Translational Research, W.C.S. Cho, Editor. 2011, Springer Science+Business Media B.V. p. 449-481.

7. Sun, J., et al., Uncovering MicroRNA and Transcription Factor Mediated Regulatory Networks in Glioblastoma. PLoS Comput Biol, 2012. 8(7): p. e1002488.

8. Volinia, S., et al., Reprogramming of miRNA networks in cancer and leukemia. Genome Res, 2010. 20(5): p. 589-99.

9. Zhang, S., et al., Discovery of multi-dimensional modules by integrative analysis of cancer genomic data. Nucleic Acids Res, 2012. 40(19): p. 9379-91.

10. Peter, M.E., Targeting of mRNAs by multiple miRNAs: the next step. Oncogene, 2010. 29(15): p. 2161-4.

11. Cochrane, D.R., et al., MicroRNA-200c mitigates invasiveness and restores sensitivity to microtubule-targeting chemotherapeutic agents. Mol Cancer Ther, 2009. 8(5): p. 1055 - 1066.

12. Cochrane, D.R., et al., Loss of miR-200c: A Marker of Aggressiveness and Chemoresistance in Female Reproductive Cancers. J Oncol, 2010. 2010: p. 821717.

13. Mateescu, B., et al., miR-141 and miR-200a act on ovarian tumorigenesis by controlling oxidative stress response. Nat Med, 2011. 17(12): p. 1627-35.

14. Feng, B., et al., MicroRNA-200b reverses chemoresistance of docetaxel-resistant human lung adenocarcinoma cells by targeting E2F3. Cancer, 2012. 118(13): p. 3365-76.

15. Meng, F., et al., Involvement of human micro-RNA in growth and response to chemotherapy in human cholangiocarcinoma cell lines. Gastroenterology, 2006. 130(7): p. 2113-29.

16. Ali, S., et al., Gemcitabine sensitivity can be induced in pancreatic cancer cells through modulation of miR-200 and miR-21 expression by curcumin or its analogue CDF. Cancer Res, 2010. 70(9): p. 3606-17.

17. Chen, G.Q., et al., Systematic analysis of microRNA involved in resistance of the MCF-7 human breast cancer cell to doxorubicin. Med Oncol, 2010. 27(2): p. 406-15.

18. Hamano, R., et al., Overexpression of miR-200c induces chemoresistance in esophageal cancers mediated through activation of the Akt signaling pathway. Clin Cancer Res, 2011. 17(9): p. 3029-38.

19. Adam, L., et al., miR-200 expression regulates epithelial-to-mesenchymal transition in bladder cancer cells and reverses resistance to epidermal growth factor receptor therapy. Clin Cancer Res, 2009. 15(16): p. 5060-72.

20. San Jose-Eneriz, E., et al., MicroRNA expression profiling in Imatinib-resistant Chronic Myeloid Leukemia patients without clinically significant ABL1-mutations. Mol Cancer, 2009. 8: p. 69.

21. Miller, T.E., et al., MicroRNA-221/222 confers tamoxifen resistance in breast cancer by targeting p27Kip1. J Biol Chem, 2008. 283(44): p. 29897-903.

22. Siddik, Z.H., Cisplatin: mode of cytotoxic action and molecular basis of resistance. Oncogene, 2003. 22(47): p. 7265-79.

23. Pommier, Y., et al., DNA topoisomerases and their poisoning by anticancer and antibacterial drugs. Chem Biol, 2010. 17(5): p. 421-33.

24. Horwitz, S.B., Taxol (paclitaxel): mechanisms of action. Ann Oncol, 1994. 5 Suppl 6: p. S3-6.

25. Alexandre, J., et al., Accumulation of hydrogen peroxide is an early and crucial step for paclitaxel-induced cancer cell death both in vitro and in vivo. Int J Cancer, 2006. 119(1): p. 418. 
26. Alexandre, J., et al., Novel action of paclitaxel against cancer cells: bystander effect mediated by reactive oxygen species. Cancer Res, 2007. 67(8): p. 3512-7.

27. Plunkett, W., et al., Gemcitabine: metabolism, mechanisms of action, and self-potentiation. Semin Oncol, 1995. 22(4 Suppl 11): p. 3-10.

28. Ohanian, M., et al., Tyrosine kinase inhibitors in acute and chronic leukemias. Expert Opin Pharmacother, 2012. 13(7): p. 927-38.

29. Yim, K.L. and D. Cunningham, Targeted drug therapies and cancer. Recent Results Cancer Res, 2011. 185: p. 159-71.

30. Rao, R.D. and M.A. Cobleigh, Adjuvant endocrine therapy for breast cancer. Oncology (Williston Park), 2012. 26(6): p. 541-7, 550, 552 passim.

31. Sun, L., et al., MiR-200b and miR-15b regulate chemotherapy-induced epithelialmesenchymal transition in human tongue cancer cells by targeting BMI1. Oncogene, 2012. 31(4): p. 432-45.

32. Gregory, P.A., et al., The miR-200 family and miR-205 regulate epithelial to mesenchymal transition by targeting ZEB1 and SIP1. Nat Cell Biol, 2008. 10(5): p. $593-601$.

33. Korpal, M., et al., The miR-200 family inhibits epithelial-mesenchymal transition and cancer cell migration by direct targeting of E-cadherin transcriptional repressors ZEB1 and ZEB2. J Biol Chem, 2008. 283(22): p. 14910 - 14914.

34. Park, S.M., et al., The miR-200 family determines the epithelial phenotype of cancer cells by targeting the E-cadherin repressors ZEB1 and ZEB2. Genes Dev, 2008. 22(7): p. 894 - 907.

35. Imanaka, Y., et al., MicroRNA-141 confers resistance to cisplatin-induced apoptosis by targeting YAP1 in human esophageal squamous cell carcinoma. J Hum Genet, 2011. 56(4): p. 270-6.

36. Marchini, S., et al., Resistance to platinum-based chemotherapy is associated with epithelial to mesenchymal transition in epithelial ovarian cancer. Eur J Cancer, 2012.

37. Pogribny, I.P., et al., Alterations of microRNAs and their targets are associated with acquired resistance of MCF-7 breast cancer cells to cisplatin. Int J Cancer, 2010. 127(8): p. 1785-94.

38. Leskela, S., et al., The miR-200 family controls beta-tubulin III expression and is associated with paclitaxel-based treatment response and progression-free survival in ovarian cancer patients. Endocr Relat Cancer, 2011. 18(1): p. 85-95.

39. Rui, W., et al., Identification of microRNA profiles in docetaxel-resistant human non-small cell lung carcinoma cells (SPC-A1). J Cell Mol Med, 2010. 14(1-2): p. 206-14.

40. Tryndyak, V.P., F.A. Beland, and I.P. Pogribny, E-cadherin transcriptional down-regulation by epigenetic and microRNA-200 family alterations is related to mesenchymal and drug-resistant phenotypes in human breast cancer cells. Int J Cancer, 2010. 126(11): p. 2575-83.

41. Kovalchuk, O., et al., Involvement of microRNA-451 in resistance of the MCF-7 breast cancer cells to chemotherapeutic drug doxorubicin. Mol Cancer Ther, 2008. 7(7): p. 2152-9.

42. Guo, L., et al., Gene expression profiling of drug-resistant small cell lung cancer cells by combining microRNA and cDNA expression analysis. Eur J Cancer, 2010. 46(9): p. 1692-702.

43. Li, Y., et al., Up-regulation of miR-200 and let-7 by natural agents leads to the reversal of epithelial-to-mesenchymal transition in gemcitabine-resistant pancreatic cancer cells. Cancer Res, 2009. 69(16): p. 6704-12.

44. Voulgari, A. and A. Pintzas, Epithelial-mesenchymal transition in cancer metastasis: mechanisms, markers and strategies to overcome drug resistance in the clinic. Biochim Biophys Acta, 2009. 1796(2): p. 75-90.

45. Strano, S., et al., The transcriptional coactivator Yes-associated protein drives $p 73$ genetarget specificity in response to DNA Damage. Mol Cell, 2005. 18(4): p. 447-59.

46. DeNicola, G.M., et al., Oncogene-induced Nrf2 transcription promotes ROS detoxification and tumorigenesis. Nature, 2011. 475(7354): p. 106-9.

47. Tian, H., et al., Keap1: one stone kills three birds Nrf2, IKKbeta and Bcl-2/Bcl-xL. Cancer Lett, 2012. 325(1): p. 26-34. 
48. Lee, D.F., et al., KEAP1 E3 ligase-mediated downregulation of NF-kappaB signaling by targeting IKKbeta. Mol Cell, 2009. 36(1): p. 131-40.

49. Kim, J.E., et al., Suppression of NF-kappaB signaling by KEAP1 regulation of IKKbeta activity through autophagic degradation and inhibition of phosphorylation. Cell Signal, 2010. 22(11): p. $1645-54$

50. Niture, S.K. and A.K. Jaiswal, Inhibitor of Nrf2 (INrf2 or Keap1) protein degrades Bcl-xL via phosphoglycerate mutase 5 and controls cellular apoptosis. J Biol Chem, 2011. 286(52): p. 44542-56.

51. Huang, Y. and W. Fan, IkappaB kinase activation is involved in regulation of paclitaxelinduced apoptosis in human tumor cell lines. Mol Pharmacol, 2002. 61(1): p. 105-13.

52. Dziadyk, J.M., et al., Paclitaxel-induced apoptosis may occur without a prior G2/M-phase arrest. Anticancer Res, 2004. 24(1): p. 27-36.

53. Bian, X., et al., NF-kappa B activation mediates doxorubicin-induced cell death in N-type neuroblastoma cells. J Biol Chem, 2001. 276(52): p. 48921-9.

54. Aggarwal, B.B., et al., Curcumin suppresses the paclitaxel-induced nuclear factor-kappaB pathway in breast cancer cells and inhibits lung metastasis of human breast cancer in nude mice. Clin Cancer Res, 2005. 11(20): p. 7490-8.

55. Kim, T., et al., $p 53$ regulates epithelial-mesenchymal transition through microRNAs targeting ZEB1 and ZEB2. J Exp Med, 2011. 208(5): p. 875-83.

56. Magenta, A., et al., miR-200c is upregulated by oxidative stress and induces endothelial cell apoptosis and senescence via ZEB1 inhibition. Cell Death Differ, 2011.

57. Neveu, P., et al., MicroRNA profiling reveals two distinct p53-related human pluripotent stem cell states. Cell Stem Cell, 2010. 7(6): p. 671-81.

58. Cummins, J.M., et al., The colorectal microRNAome. Proc Natl Acad Sci U S A, 2006. 103(10): p. 3687-92.

59. Ostling, P., et al., Systematic analysis of microRNAs targeting the androgen receptor in prostate cancer cells. Cancer Res, 2011. 71(5): p. 1956-67.

60. Helzlsouer, K.J., et al., Serum gonadotropins and steroid hormones and the development of ovarian cancer. JAMA, 1995. 274(24): p. 1926-30.

61. Ilekis, J.V., et al., Expression of epidermal growth factor and androgen receptors in ovarian cancer. Gynecol Oncol, 1997. 66(2): p. 250-4.

62. Marchion, D.C., et al., BAD phosphorylation determines ovarian cancer chemosensitivity and patient survival. Clin Cancer Res, 2011. 17(19): p. 6356-66.

63. Ayantunde, A.A. and S.L. Parsons, Pattern and prognostic factors in patients with malignant ascites: a retrospective study. Ann Oncol, 2007. 18(5): p. 945-9.

64. Iorio, M.V. and C.M. Croce, MicroRNA dysregulation in cancer: diagnostics, monitoring and therapeutics. A comprehensive review. EMBO Mol Med, 2012. 4(3): p. 143-59.

65. Lindow, M. and S. Kauppinen, Discovering the first microRNA-targeted drug. J Cell Biol, 2012. 199(3): p. 407-12.

66. Krutzfeldt, J., et al., Silencing of microRNAs in vivo with 'antagomirs'. Nature, 2005. 438(7068): p. 685-9.

67. Elmen, J., et al., LNA-mediated microRNA silencing in non-human primates. Nature, 2008. 452(7189): p. 896-9.

68. Ebert, M.S., J.R. Neilson, and P.A. Sharp, MicroRNA sponges: competitive inhibitors of small RNAs in mammalian cells. Nat Methods, 2007. 4(9): p. 721-6.

69. Haraguchi, T., Y. Ozaki, and H. Iba, Vectors expressing efficient RNA decoys achieve the longterm suppression of specific microRNA activity in mammalian cells. Nucleic Acids Res, 2009. 37(6): p. e43.

70. Esquela-Kerscher, A., et al., The let-7 microRNA reduces tumor growth in mouse models of lung cancer. Cell Cycle, 2008. 7(6): p. 759 - 64.

71. Kumar, M.S., et al., Suppression of non-small cell lung tumor development by the let-7 microRNA family. Proc Natl Acad Sci U S A, 2008. 105(10): p. 3903 - 8. 
72. Kota, J., et al., Therapeutic microRNA delivery suppresses tumorigenesis in a murine liver cancer model. Cell, 2009. 137(6): p. 1005 - 17.

73. Wiggins, J.F., et al., Development of a lung cancer therapeutic based on the tumor suppressor microRNA-34. Cancer Res, 2010. 70(14): p. 5923-30.

74. Park, J.K., et al., miR-221 silencing blocks hepatocellular carcinoma and promotes survival. Cancer Res, 2011. 71(24): p. 7608-16.

75. Ma, L., et al., Therapeutic silencing of miR-10b inhibits metastasis in a mouse mammary tumor model. Nat Biotechnol, 2010. 28(4): p. 341-7.

76. Calin, G.A., et al., MiR-15a and miR-16-1 cluster functions in human leukemia. Proc Natl Acad Sci U S A, 2008. 105(13): p. 5166-71.

77. Si, M.L., et al., miR-21-mediated tumor growth. Oncogene, 2007. 26(19): p. 2799-803.

78. Leucci, E., et al., Inhibition of miR-9 de-represses HUR and DICER1 and impairs Hodgkin lymphoma tumour outgrowth in vivo. Oncogene, 2012.

79. Swarbrick, A., et al., miR-380-5p represses $p 53$ to control cellular survival and is associated with poor outcome in MYCN-amplified neuroblastoma. Nat Med, 2010. 16(10): p. 1134-40.

80. Wurdinger, T., et al., miR-296 regulates growth factor receptor overexpression in angiogenic endothelial cells. Cancer Cell, 2008. 14(5): p. 382-93.

81. Tazawa, H., et al., Tumor-suppressive miR-34a induces senescence-like growth arrest through modulation of the E2F pathway in human colon cancer cells. Proc Natl Acad Sci U S A, 2007. 104(39): p. 15472-7.

82. Chen, Y., et al., Nanoparticles modified with tumor-targeting SCFv deliver siRNA and miRNA for cancer therapy. Mol Ther, 2010. 18(9): p. 1650-6.

83. Su, J., et al., Silencing microRNA by interfering nanoparticles in mice. Nucleic Acids Res, 2011. 39(6): p. e38.

84. Babar, I.A., et al., Nanoparticle-based therapy in an in vivo microRNA-155 (miR-155)dependent mouse model of lymphoma. Proc Natl Acad Sci U S A, 2012. 109(26): p. E1695-704.

85. Trang, P., et al., Regression of murine lung tumors by the let-7 microRNA. Oncogene, 2010. 29(11): p. 1580-7.

86. Liu, C., et al., The microRNA miR-34a inhibits prostate cancer stem cells and metastasis by directly repressing CD44. Nat Med, 2011. 17(2): p. 211-5.

87. Pramanik, D., et al., Restitution of tumor suppressor microRNAs using a systemic nanovector inhibits pancreatic cancer growth in mice. Mol Cancer Ther, 2011. 10(8): p. 1470-80.

88. Kim, J., et al., High-grade serous ovarian cancer arises from fallopian tube in a mouse model. Proc Natl Acad Sci U S A, 2012. 109(10): p. 3921-6.

89. Shan, W. and J. Liu, Epithelial ovarian cancer: focus on genetics and animal models. Cell Cycle, 2009. 8(5): p. 731-5.

90. Gentner, B., et al., Stable knockdown of microRNA in vivo by lentiviral vectors. Nat Methods, 2009. 6(1): p. 63-6.

91. Michelfelder, S. and M. Trepel, Adeno-associated viral vectors and their redirection to celltype specific receptors. Adv Genet, 2009. 67: p. 29-60.

92. Cronin, J., X.Y. Zhang, and J. Reiser, Altering the tropism of lentiviral vectors through pseudotyping. Curr Gene Ther, 2005. 5(4): p. 387-98.

93. Sudimack, J. and R.J. Lee, Targeted drug delivery via the folate receptor. Adv Drug Deliv Rev, 2000. 41(2): p. 147-62.

94. Karst, A.M. and R. Drapkin, The new face of ovarian cancer modeling: better prospects for detection and treatment. F1000 Med Rep, 2011. 3: p. 22. 